\title{
Mathematical Foundations for the Boundary- Field Equation Methods in Acoustic and Electromagnetic Scattering
}

\author{
George C. Hsiao *
}

\begin{abstract}
The essence of the boundary-field equation method is the reduction of the boundary value problem under consideration to an equivalent nonlocal boundary value problem in a bounded domain by using boundary integral equations. The latter can then be treated by the standard variational method including its numerical approximations. In this paper, various formulations of the nonlocal boundary value problems will be given for the Helmholtz equation as well as for the time- harmonic Maxwell equations. Emphasis will be placed upon the variational formulation for the method and mathematical foundations for the solution procedure. Some numerical experiments are included for a model problem in electromagnetic scattering.
\end{abstract}

\section{Introduction-basic concepts}

The idea of combining the boundary integral equation method with other techniques had already been introduced in a paper by Greenspan and Werner [6] in the study of an exterior Dirichlet problem for the Helmholtz equation in 2-D. Specially, they employed a combined boundary integral equation and the finite difference methods in a bounded domain in order to obtain sufficiently accurate approximate solutions at points near the boundary. Alternatively, one may also consider combining the boundary integral equation method with other methods such as the finite element method as in the paper by MacCamy and Marin [11] for exterior interface problems. In general we refer to a variational method for treating boundary value problems for partial differential equations in a bounded domain together with some nonlocal boundary conditions in the form of boundary integral equations as the boundary-field equation method (see, e.g., the monograph by Gatica and Hsiao [5]).

For the exterior boundary value problem (BVP), the general solution procedure for the method is shown in Figure 1. Of course for the scattering problem we may

\footnotetext{
*Department of Mathematical Sciences, University of Delaware, Newark, Delaware 19716.
} 
start directly with the transmission problem. In this paper we will confine to the steps concerning the various nonlocal BVPs and their corresponding weak formulations in acoustics as well as in electromagnetics.

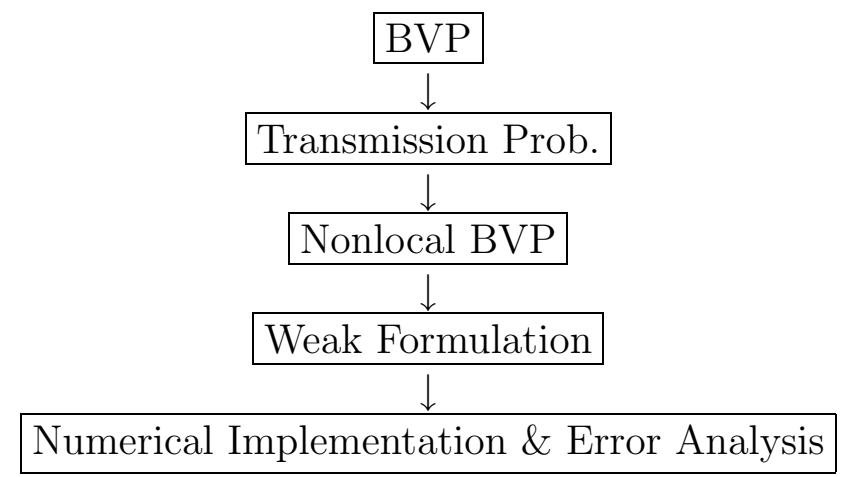

Figure 1: A flow chart for the general solution procedure.

As will be seen, for the transmission problem, the nonlocal boundary condition will be derived from boundary integral equation methods and it will give the relation among the Cauchy data of the solution on the interface boundary. It is important to point out that in general the process of the reduction from a BVP to boundary integral equation formulations is not unique; hence one may arrive at various different forms of nonlocal boundary value problems. However, in all the cases, one tries to obtain an equivalent nonlocal boundary value problem whose weak formulation will provide a bilinear form to satisfy a Gårding inequality in appropriate function space. In fact, the Gårding inequality is one of the main mathematical ingredients for the variational methods. It is well known that Gårding's inequality implies the validity of the Fredholm alternatives for the variational equations under consideration. If in addition the finite-dimensional approximate subspaces approximate the solution space in the energy norm, then the Babǔska-Brezzi-Ladzenskaya condition (the so-called $B B L$-condition) follows also from Gårding's inequality together with the uniqueness of the solution (see, e.g., [13]). This BBL-condition is a crucial stability condition for the Galerkin method. As a consequence, one has the celebrated Ceá's lemma which implies that the Galerkin solution is the best approximation for the variational solution in the energy norm. In fact, from Ceá's lemma, one reduces the problem of error estimates to a problem in approximation theory. Together with approximation and inverse properties of the corresponding finite dimensional approximate function space, qusioptimal error estimates for the Galerkin solution can be established by using standard arguments in functional analysis (see, e.g., [15]). 


\section{Acoustic Scattering}

Through out the paper, let $\Omega$ be a bounded domain in $\mathbb{R}^{3}$ with smooth boundary $\Gamma$. We consider the exterior BVP for the Helmholtz equation

$$
\begin{gathered}
\Delta u+k^{2} u=0 \text { in } \Omega^{c}:=\mathbb{R}^{3} \backslash \bar{\Omega}, \quad u=0 \quad \text { on } \quad \Gamma, \\
\frac{\partial u^{s}}{\partial r}-i k u^{s}=o\left(r^{-1}\right) \quad \text { as } \quad r=|x| \rightarrow \infty,
\end{gathered}
$$

where $u=u^{s}+u^{i}$ denotes the total field, $u^{s}$ the unknown scattered field, and $u^{i}$ the given incident field. For simplicity, we assume that the wave number $k$ is a constant and that $\operatorname{Im} k \geq 0$ in order to avoid resonance states. The Dirichlet problem (1), (2) is one of the basic problems in classical scattering theory. It represents the scattering of time-harmonic acoustic waves by a bounded impenetrable obstacle $\Omega$ and the homogeneous Dirichlet boundary condition in (1) corresponds to a soundsoft obstacle. The Sommerfeld radiation condition (2) guarantees the scattered wave $u^{s}$ is outgoing and will ensure uniqueness for the solutions to the scattering problem.

We now reformulate the BVP as a transmission problem. We divide the domain $\Omega^{c}$ into two subdomains $\Omega_{F}$ and $\Omega_{B}=\Omega^{c} \backslash \bar{\Omega}_{F}$ by introducing an artificial common boundary $\Gamma_{0}$ so that $\Omega$ is completely contained in $\Gamma_{0}$. Then (1), (2) may be reformulated as a transmission problem

$$
\begin{gathered}
\Delta u+k^{2} u=0 \quad \text { in } \quad \Omega_{F} ; \quad u=0 \quad \text { on } \quad \Gamma, \\
u^{-}=u^{s+}+u^{i}, \quad \frac{\partial u^{-}}{\partial n}=\frac{\partial u^{s+}}{\partial n}+\frac{\partial u^{i}}{\partial n} \quad \text { on } \Gamma_{0}, \\
\Delta u^{s}+k^{2} u^{s}=0 \quad \text { in } \quad \Omega_{B} \\
\frac{\partial u^{s}}{\partial r}-i k u^{s}=o\left(r^{-1}\right) \quad \text { as } \quad r=|x| \longrightarrow \infty .
\end{gathered}
$$

In the formulation, it is understood that the given incident field $u^{i}$ is required also to satisfy the Helmholtz equation in $\Omega_{B}$. Here and in the sequel, we have adapted the notation $v^{-}$and $v^{+}$for the limits of $\mathrm{v}$ on $\Gamma_{0}$ from $\Omega_{F}$ and $\Omega_{B}$, respectively, and $\partial / \partial n$ for the normal derivative with respect to the unit outward normal $n$ to $\Gamma_{0}$.

To reduce the transmission problem $(3),(4),(5)$ to a nonlocal boundary value problem, we begin with the Green representation for $u^{s}$ in $\Omega_{B}$,

$$
u^{s}(x)=\int_{\Gamma_{0}} u^{s}(y)^{+} \frac{\partial}{\partial n_{y}} \gamma(x, y) d s_{y}-\int_{\Gamma_{0}} \frac{\partial}{\partial n_{y}} u^{s}(y)^{+} \gamma(x, y) d s_{y}, \quad x \in \Omega_{B},
$$

where $\gamma(x, y):=e^{i k|x-y|} / 4 \pi|x-y|$, is the fundamental solution for the Helmholtz equation. In the representation, the functions $u^{s}(y)^{+}$and $\frac{\partial}{\partial n_{y}} u^{s}(y)^{+}$are unknown 
on the interface $\Gamma_{0}$. These are the Cauchy data of the solution $u^{s}$ to the Helmholtz equation in $\Omega_{B}$ and they are related by the two boundary integral equations (BIEs)

$$
\left(\begin{array}{c}
u^{s+} \\
\frac{\partial u^{s+}}{\partial n}
\end{array}\right)=\left(\begin{array}{cc}
\frac{1}{2} I+K & -V \\
-W & \frac{1}{2} I-K^{\prime}
\end{array}\right)\left(\begin{array}{c}
u^{s+} \\
\frac{\partial u^{s+}}{\partial n}
\end{array}\right) \text { on } \Gamma_{0} .
$$

Here $V, K, K, W$ are the four basic boundary integral operators defined by

$$
\begin{array}{rlrl}
V \sigma(x) & :=\int_{\Gamma_{0}} \gamma(x, y) \sigma(y) d s_{y} & , K \mu(x) & :=\int_{\Gamma_{0}} \frac{\partial}{\partial n_{y}} \mu(y) \gamma(x, y) d s_{y} \\
K^{\prime} \sigma(x) & :=\int_{\Gamma_{0}} \frac{\partial}{\partial n_{x}} \sigma(y) \gamma(x, y) d s_{y} \quad, \quad W \mu(x):=-\frac{\partial}{\partial n_{x}} \int_{\Gamma_{0}} \frac{\partial}{\partial n_{y}} \mu(y) \gamma(x, y) d s_{y},
\end{array}
$$

while the matrix of boundary integral operators

$$
\mathcal{C}_{\Omega_{B}}:=\left(\begin{array}{cc}
\frac{1}{2} I+K & -V \\
-W & \frac{1}{2} I-K^{\prime}
\end{array}\right)
$$

is referred to as the Calderon projector which maps the Cauchy data into itself. We note that the solution $u^{s}$ in the exterior domain $\Omega_{B}$ is completely determined from the representation (6), provided one knows its Cauchy data on the interface $\Gamma_{0}$. This leads us to the consideration of various nonlocal boundary value problems which all will reduce the transmission problem (3),(4),(5) to an equivalent problem in the bounded domain $\Omega_{F}$.

If we denote $\sigma=\partial u^{s+} / \partial n$, and use the first BIE in (7), we arrive at the simplest nonlocal boundary value problem: Find $(u, \sigma)$ such that

$$
\begin{gathered}
\Delta u+k^{2} u=0 \quad \Omega_{\mathrm{F}} ; \quad u=0 \quad \text { on } \quad \Gamma, \\
\frac{\partial u^{-}}{\partial n}=\sigma+\frac{\partial u^{i}}{\partial n} \quad \text { on } \quad \Gamma_{0}, \\
V \sigma+\left(\frac{1}{2} I-K\right)\left(u^{-}-u^{i}\right)=0 \quad \text { on } \Gamma_{0} .
\end{gathered}
$$

The boundary condition (10) is nonlocal in the sense that to find $\sigma$ at a single point on $\Gamma_{0}$, one needs the values of $u^{-}$at every point on $\Gamma_{0}$. In some sense (10) serves as a boundary integral equation of the first kind for the unknown $\sigma$ by considering $u^{-}$ as well as $u^{i}$ as the given data. We note that if $\sigma$ is given on $\Gamma_{0}$, then (8) and (9) will be sufficient for determining $u$ in $\Omega_{F}$. Alternatively, we may use both BIEs in (7) to formulate the nonlocal boundary value problem. More precisely, one may replace (9) by

$$
\frac{\partial u^{-}}{\partial n}=-W\left(u^{-}-u^{i}\right)+\left(\frac{1}{2} I-K^{\prime}\right) \sigma+\frac{\partial u^{i}}{\partial n} \quad \text { on } \quad \Gamma_{0} .
$$


Then the problem consisting of (8),(9a),(10) is an another version of nonlocal boundary value problem for $(u, \sigma)$. This is the symmetric version as can be seen from its variational formulation [4], and it does not depend on the compactness of the operator $K$ as in the one BIE formulation, (8),(9),(10).

For the variational formulation, we need the energy spaces:

$$
H_{\Gamma}^{1}\left(\Omega_{F}\right):=\left\{v \in H^{1}\left(\Omega_{F}\right): v_{\mid \Gamma}=0\right\}, \quad H^{-\frac{1}{2}}\left(\Gamma_{0}\right)=\text { dual of } H^{\frac{1}{2}}\left(\Gamma_{0}\right) .
$$

Now the variational formulation for the nonolocal boundary value problem (8),(9),(10) reads: Find $(u, \sigma) \in H_{\Gamma}^{1}\left(\Omega_{F}\right) \times H^{\frac{1}{2}}\left(\Gamma_{0}\right)$ such that

$$
\begin{gathered}
a(u, v)-<\sigma, v^{-}>=<\frac{\partial u^{i}}{\partial n}, v^{-}> \\
<\chi, V \sigma>+<\chi,\left(\frac{1}{2} I-K\right) u^{-}>=<\chi,\left(\frac{1}{2} I-K\right) u^{i}>
\end{gathered}
$$

for all $(v, \chi) \in H_{\Gamma}^{1}\left(\Omega_{F}\right) \times H^{\frac{1}{2}}\left(\Gamma_{0}\right)$, where

$$
a(u, v):=\int_{\Omega_{F}}\left\{\nabla u \overline{\nabla v}-k^{2} u \bar{v}\right\} d x
$$

and $<\cdot, \cdot>$ denotes the duality pairing between $H^{-1 / 2}\left(\Gamma_{0}\right)$ and $H^{1 / 2}\left(\Gamma_{0}\right)$.

For the problem $(8),(9 \mathrm{a}),(10)$, the variational equational (11) will be replaced by

$$
a(u, v)+<W u^{-}-\left(\frac{1}{2} I-K^{\prime}\right) \sigma, v^{-}>=<\frac{\partial u^{i}}{\partial n}+W u^{i}, v^{-}>
$$

because of (9a), but (12) remains unchanged.

The following results can now be established (see, e.g. [7]).

Theorem 2.1 The sesquilinear form $A_{k}(\cdot, \cdot), k=1,2$, from the variational problems (11),(12), and (11a), (12) defined by

$$
\begin{gathered}
A_{1}((u, \sigma),(v, \chi)):=a(u, \sigma)-<\sigma, v^{-}>+2\left\{<\chi, V \sigma>+<\chi,\left(\frac{1}{2} I-K\right) u^{-}>\right\}, \\
A_{2}((u, \sigma),(v, \chi)):=a(u, \sigma)+<W u^{-}, v^{-}>-<\left(\frac{1}{2} I-K^{\prime}\right) \sigma, v^{-}> \\
+<\chi, V \sigma>+<\chi,\left(\frac{1}{2} I-K\right) u^{-}>
\end{gathered}
$$

respectively, satisfies a Gårding inequality in the form:

$$
\operatorname{Re} A_{k}((v, \chi),(v, \chi)) \geq \alpha_{k}||(v, \chi) \|_{\mathcal{E}}^{2}-\left|C_{k}((v, \chi),(v, \chi))\right| \quad \text { for all } \quad(v, \chi) \in \mathcal{E},
$$

where $\alpha_{k}>0$ is a constant, $C_{k}$ is a compact form on $\mathcal{E}:=H_{\Gamma}^{1}\left(\Omega_{F}\right) \times H^{\frac{1}{2}}\left(\Gamma_{0}\right)$, and $\|(v, \chi)\|_{\mathcal{E}}^{2}:=\|v\|_{H_{\Gamma}^{1}\left(\Omega_{F}\right)}^{2}+\|\chi\|_{H^{\frac{1}{2}\left(\Gamma_{0}\right)}}^{2}$. 


\section{Electromagnetic Scattering}

We now consider the scattering of time-harmonic electromagnetic waves by an obstacle surrounded by a homogeneous medium with constant electric permittivity $\epsilon$ and magnetic permeability $\mu$. This leads us to the exterior BVP for the reduced Maxwell equations:

$$
\begin{gathered}
\nabla \times E=i \omega \mu H ; \nabla \times H=-i \omega \epsilon E \quad \text { in } \quad \Omega^{c}:=\mathbb{R}^{3} \backslash \bar{\Omega} \\
n \times E=0 \quad \text { on } \quad \Gamma, \\
\lim _{|x| \rightarrow \infty}\left(\sqrt{\mu} H^{s} \times \frac{x}{|x|}-\sqrt{\epsilon} E^{s}\right)=0 \\
E=E^{s}+E^{i} ; \quad H=H^{s}+H^{i} .
\end{gathered}
$$

In the formulation, $E$ and $H$ are, respectively, the electric and magnetic fields of the total electromagnetic wave with frequency $\omega>0$. We assume again that $\operatorname{Im} k \geq 0$, where the wave number $k$ is a constant given by $k^{2}=\epsilon \mu \omega^{2}$. The boundary condition (14) simply implies that we have a perfectly conducting obstacle $\Omega$, where $n$ is the unit outward normal to the boundary $\Gamma$. The Silver-Müller radiation condition (15) is the counterpart of the Sommerfeld radiation condition (2) for elctromagnetic waves.

Let $\Gamma_{0}$ be the artificial boundary as in the acoustic scattering. In the same manner, we may convert the BVP (13)-(16) to a transmission problem of the form,

$$
\begin{gathered}
\nabla \times E=i \omega \mu ; \nabla \times H=-i \omega \epsilon E \text { in } \Omega_{F}, \\
n \times E=0 \quad \text { on } \Gamma, \\
n \times E=n \times E^{s}+n \times E^{i} ; \quad H \times n=H^{s} \times n+H^{i} \times n \text { on } \Gamma_{0}, \\
\nabla \times E^{s}=i \omega \mu H^{s} ; \nabla \times H^{s}=-i \omega \epsilon E^{s} \text { in } \Omega_{B}, \\
\lim _{|x| \rightarrow \infty}\left(\sqrt{\mu} H^{s} \times \frac{x}{|x|}-\sqrt{\epsilon} E^{s}\right)=0 .
\end{gathered}
$$

Now in order to reduce the transmission (17)-(21) to a nonlocal boundary value problem, we begin with the well known Stratton-Chu formulas for $E^{s}$ and $H^{s}$ in [14],

$$
\begin{aligned}
E^{s}(x) & =-i \omega \mu \int_{\Gamma_{0}} \gamma(x, y)\left(H^{s} \times n\right) d s_{y}+\int_{\Gamma_{0}}\left(n \cdot E^{s}\right) \nabla_{y} \gamma(x, y) d s_{y} \\
& -\int_{\Gamma_{0}} \nabla_{y} \gamma(x, y) \times\left(n \times E^{s}\right) d s_{y}, \quad x \in \Omega_{B}, \\
H^{s}(x) & =-i \omega \epsilon \int_{\Gamma_{0}} \gamma(x, y)\left(n \times E^{s}\right)+\int_{\Gamma_{0}}\left(n \cdot H^{s}\right) \nabla_{y} \gamma(x, y) d s_{y} \\
& +\int_{\Gamma_{0}} \nabla_{y} \gamma(x, y) \times\left(H^{s} \times n\right) d s_{y}, \quad x \in \Omega_{B},
\end{aligned}
$$

where $\gamma(x, y)$ is again the fundamental solution for the Helmholtz equation (see (6)). In some way, the Stratton-Chu formulas are misleading from the mathematical point 
of view. We see that the representation formulas require more Cauchy data on interface $\Gamma_{0}$ than those appeared in (19). This is due to the fact that the formulas were derived by using only the fundamental solution of the Helmholtz equation. In fact, from the Maxwell equations, one can easily show that the additional boundary data can be eliminated (see, e.g., [8]), and we will obtain a modified version of the Stratton-Chu formulas for $E^{s}$ and $H^{s}$,

$$
\begin{aligned}
E^{s}(x)= & -i \omega \mu \int_{\Gamma_{0}}\left\{\gamma(x, y)-\frac{1}{k^{2}} \nabla_{y} \gamma(x, y) d i v_{\Gamma_{0}}\right\}\left(H^{s} \times n\right) d s_{y} \\
& -\int_{\Gamma_{0}} \nabla_{y} \gamma(x, y) \times\left(n \times E^{s}\right) d s_{y}, \quad x \in \Omega_{B}, \\
H^{s}(x)= & -i \omega \epsilon \int_{\Gamma_{0}}\left\{\gamma(x, y)-\frac{1}{k^{2}} \nabla_{y} \gamma(x, y) d i v_{\Gamma_{0}}\right\}\left(n \times E^{s}\right) d s_{y} \\
& +\int_{\Gamma_{0}} \nabla_{y} \gamma(x, y) \times\left(H^{s} \times n\right) d s_{y}, \quad x \in \Omega_{B} .
\end{aligned}
$$

Here $H^{s} \times n$ and $n \times E^{s}$ are the appropriate Cauchy data. They are related by the BIEs

$$
\left(\begin{array}{c}
n \times E^{s} \\
H^{s} \times n
\end{array}\right)=\left(\begin{array}{cc}
\frac{1}{2} I-R & -i \omega \mu T \\
i \omega \epsilon T & \frac{1}{2} I-R
\end{array}\right)\left(\begin{array}{c}
n \times E^{s} \\
H^{s} \times n,
\end{array}\right) \text { on } \Gamma_{0},
$$

where the boundary integral operators are defined by

$$
\begin{gathered}
T(\varphi)(x):=n \times \int_{\Gamma_{0}}\left\{\gamma(x, y)-\frac{1}{k^{2}} \nabla_{y} \gamma(x, y) d i v_{\Gamma_{0}}\right\} \varphi(y) d s_{y}, \\
R(\varphi)(x):=n \times \int_{\Gamma_{0}} \nabla_{y} \gamma(x, y) \times \varphi(y) d s_{y} .
\end{gathered}
$$

In an analogue to (7), the Calderon projector defined by (24) maps the Cauchy data $j:=H^{s} \times n, m:=n \times E^{s}$ into itself. These are the electric current of density $j$, and the magnetic current of density $m$. We refer to the first and the second BIEs in (24) as the EFIE and MFIE respectively, since from these equations, we may recover the conventional electric and magnetic field integral equations, if $\Omega_{F}$ is a perfect conductor.

We may now formulate the nonlocal BVP by using the BIEs from (24). For one BIE formulation based on the EFIE, we have the formulation: Find $(E, H, j)$ such that

$$
\begin{gathered}
\nabla \times E=i \omega \mu H ; \quad \nabla \times H=-i \omega \epsilon E \text { in } \Omega_{F}, \\
n \times E=0 \quad \text { on } \Gamma, \\
H \times n=j+H^{i} \times n \text { on } \Gamma_{0},
\end{gathered}
$$




$$
-i \omega \mu T j-\left(\frac{1}{2} I+R\right)\left(n \times E-n \times E^{i}\right)=0 \quad \text { on } \quad \Gamma_{0} .
$$

We remark that in principle one may also replace the nonlocal boundary condition (28) by using the MFIE (see Section 4 ). Moreover, we may apply the $\nabla \times$ to the Maxwell equations in order to eliminate one of the unknowns. This leads to the formulation: Find $(E, j)$ such that

$$
\begin{gathered}
\nabla \times \nabla \times E-k^{2} E=0 \quad \text { in } \Omega_{F}, \\
n \times E=0 \quad \text { on } \Gamma, \\
(1 / i \omega \mu) n \times(\nabla \times E)=-\left(j+H^{i} \times n\right) \text { on } \Gamma_{0}
\end{gathered}
$$

together with (28), where in (27a), $j$ is replaced by

$$
j=\left(\frac{1}{2} I-R\right) j+i \omega \epsilon T\left(n \times E-n \times E^{i}\right) \text { on } \Gamma_{0} .
$$

We note that (27a) is the same as (27) in view of (25) and that (27b) is the MFIE in (24). This formulation based on the second order partial differential equation (25a) together with (26), (27a) with (27b), and (28) is in some sense closest to the formulations for the elliptic BVPs as in the acoustic scattering. It has been analysesed recently by Ammari and Nédélec in [1]. In the following, we will confine ourselves to the variational formulation for this nonlocal boundary value problem. We remark that one may also replace (25) by (25a) in the previous formulation which will be resemble to the one BIE formulation in the acoustic scattering.

To consider the variational formulation for the two BIE nonlocal boundary value problem, we need the appropriate Sobolev spaces. In fact, as well be seen, in contrast to the acoustic case, the mathematical analysis here is not so transparent. To motive our observation, let us begin with the weak form of (25a) subject to the conditions (26) and (27a),

$$
b(E, \tilde{E})-i \omega \int_{\Gamma_{0}} j \cdot \overline{\tilde{E}} d s=i \omega \int_{\Gamma_{0}}\left(H^{i} \times n\right) \cdot \overline{\tilde{E}} d s
$$

where $b(E, \tilde{E})$ is the sesqulinear form associated with (25a) defined by

$$
b(E, \tilde{E}):=\int_{\Omega_{F}} \frac{1}{\mu}(\nabla \times E) \cdot(\nabla \times \overline{\tilde{E}}) d x-\omega^{2} \int_{\Omega_{F}} \epsilon E \cdot \overline{\tilde{E}} d x .
$$

The latter suggests that the proper solution space for $E$ (also the test function space for $\tilde{E})$ should be a subspace of $H\left(\operatorname{curl}, \Omega_{F}\right):=\left\{v \in\left(L^{2}\left(\Omega_{F}\right)\right)^{3}: \nabla \times v \in\left(L^{2}\left(\Omega_{F}\right)\right)^{3}\right\}$, that is,

$$
H_{0}\left(\operatorname{curl}, \Omega_{F}\right):=\left\{v \in H\left(\operatorname{curl}, \Omega_{F}\right): n \times\left. v\right|_{\Gamma}=0\right\} .
$$


Then from (29), we see that if the magnetic field $H$ is also in $H\left(\operatorname{curl}, \Omega_{F}\right)$, then $j$ must be in the trace space,

$$
H_{d i v}^{-1 / 2}\left(\Gamma_{0}\right):=\left\{v \in T H^{-1 / 2}\left(\Gamma_{0}\right): \operatorname{div}_{\Gamma_{0}} v \in H^{-1 / 2}\left(\Gamma_{0}\right)\right\}
$$

The latter can be naturally identified with the dual of the trace space

$$
H_{\text {curl }^{*}}^{-1 / 2}\left(\Gamma_{0}\right):=\left\{v \in T H^{-1 / 2}\left(\Gamma_{0}\right): \operatorname{curl}_{\Gamma_{0}}^{*} v \in H^{-1 / 2}\left(\Gamma_{0}\right)\right\} .
$$

Here we denote the tangential space by $T H^{s}\left(\Gamma_{0}\right):=\left\{v \in\left(H^{s}\left(\Gamma_{0}\right)\right)^{3}:\left.v \cdot n\right|_{\Gamma_{0}}=0\right\}$. We denote by $\nabla_{\Gamma_{0}}$ the surface gradient, and define $\operatorname{div}_{\Gamma_{0}}:=\nabla_{\Gamma_{0}} \cdot$ curl $_{\Gamma_{0}}:=n \times \nabla_{\Gamma_{0}}$, and $-\operatorname{curl}_{\Gamma_{0}}^{*}:=$ the transpose of $\operatorname{curl}_{\Gamma_{0}}$. We now have the variational formulation for the nonlocal BVP consisting of (25a),(26),(27a),(27b) and (28): Find $(E, j) \in$ $H_{0}\left(\right.$ curl,$\left.\Omega_{F}\right) \times H_{d i v}^{-1 / 2}\left(\Gamma_{0}\right)$ satisfying

$$
\begin{gathered}
b(E, \tilde{E})-i \omega \int_{\Gamma_{0}} j^{*} \cdot \overline{\tilde{E}} d s=i \omega \int_{\Gamma_{0}}\left(H^{i n c} \times n\right) \cdot \overline{\tilde{E}} d s, \quad \text { and } \\
-i \omega \mu \int_{\Gamma_{0}} T j \cdot \overline{\tilde{j}} d s-\int_{\Gamma_{0}}\left(\frac{1}{2} I-R\right)(n \times E) \cdot \overline{\tilde{j}} d s=-\int_{\Gamma_{0}}\left(\frac{1}{2} I-R\right)\left(n \times E^{i}\right) \cdot \overline{\tilde{j}} d s
\end{gathered}
$$

for all $(\tilde{E}, \tilde{j}) \in H_{0}\left(\operatorname{curl}, \Omega_{F}\right) \times H_{\text {div }}^{-1 / 2}\left(\Gamma_{0}\right)$, where in $(31)$

$$
j^{*}:=\left(\frac{1}{2} I-R\right) j+i \omega \epsilon T\left(n \times E-n \times E^{i n c}\right) .
$$

We note that in the above variational formulation, the sesqulinear form (30) contains the $L^{2}$-term. However, in contrast to the case for the Helmholtz equation, it is known that the embedding from $H\left(\right.$ curl, $\left.\Omega_{F}\right)$ to $L^{2}\left(\Omega_{F}\right)$ is not compact. This means that we are not able to establish a Gårding inequality on the energy space $H_{0}\left(\right.$ curl,$\left.\Omega_{F}\right) \times H_{d i v}^{-1 / 2}\left(\Gamma_{0}\right)$ directly without any modifications. To overcome this difficulty, we need the following result for the decomposition of function spaces which is known as the Helmholtz or Hodge decomposition [3],[10] .

Lemma 3.1 (1) Let $M\left(\Omega_{F}\right)$ and $P\left(\Omega_{F}\right)$ be the spaces defined by

$$
\begin{gathered}
M\left(\Omega_{F}\right):=\left\{v \in H_{0}\left(\text { curl }, \Omega_{F}\right): \int_{\Omega_{F}} \epsilon v \cdot \nabla q=0, \forall q \in P\left(\Omega_{F}\right)\right\}, \quad \text { and } \\
P\left(\Omega_{F}\right):=\left\{p \in H^{1}\left(\Omega_{F}\right):\left.p\right|_{\Gamma}=0\right\} .
\end{gathered}
$$

Then the decomposition

$$
H_{0}\left(\operatorname{curl}, \Omega_{F}\right)=M\left(\Omega_{F}\right) \oplus \nabla P\left(\Omega_{F}\right)
$$

is orthogonal with respect to the inner product

$$
((u, v)):=\int_{\Omega_{F}} \nabla \times u \cdot \overline{\nabla \times v}+\int_{\Omega_{F}} \epsilon u \cdot \bar{v} .
$$


(2) The decomposition

$$
H_{d i v}^{-1 / 2}\left(\Gamma_{0}\right)=\nabla_{\Gamma_{0}} H^{3 / 2}\left(\Gamma_{0}\right) / C \oplus \operatorname{curl}_{\Gamma_{0}} H^{1 / 2}\left(\Gamma_{0}\right) / C
$$

is orthogonal with respect to the duality product between $T H^{1 / 2}\left(\Gamma_{0}\right)$ and $T H^{-1 / 2}\left(\Gamma_{0}\right)$.

We note that the integral condition in the space $M\left(\Omega_{F}\right)$ is equivalent to require that $\operatorname{div}(\epsilon v)=0$ in $\Omega_{F}$ and that $v \cdot n=0$ on $\Gamma_{0}$. Hence the embedding from $M\left(\Omega_{F}\right)$ to $L^{2}\left(\Omega_{F}\right)$ is compact. With the Hodge decomposition available, we are now in a position to discuss the Gårding inequality. The following theorem has been recently established in [1].

Theorem 3.2 In the variational formulation for $(31),(32),(33)$, let

$$
\begin{gathered}
E=u+\nabla p, u \in M\left(\Omega_{F}\right), p \in P(\Omega), \\
j=\nabla_{\Gamma_{0}} \varphi+\operatorname{curl}_{\Gamma_{0}} \psi \in H_{d v i}^{-1 / 2}\left(\Gamma_{0}\right)
\end{gathered}
$$

and denote the corresponding sesquiliear form by $\mathcal{A}((u, p, \varphi, \psi),(v, q, \sigma, \lambda))$, where $(v, q, \sigma, \lambda)$ are the test functions in

$$
\mathcal{H}:=M\left(\Omega_{F}\right) \times P\left(\Omega_{F}\right) \times H^{3 / 2}\left(\Gamma_{0}\right) / C \times H^{1 / 2}\left(\Gamma_{0}\right) / C .
$$

Then $\mathcal{A}$ satisfies the Gairding inequality:

$$
\operatorname{Re} \mathcal{A}\left((v, q, \sigma, \lambda),((v, q, \sigma, \lambda)) \geq \alpha||(v, q, \sigma, \lambda) \|_{\mathcal{H}}^{2}-|\mathcal{C}((v, q, \sigma, \lambda),(v, q, \sigma, \lambda))|\right.
$$

for all $(v, q, \sigma, \lambda) \in \mathcal{H}$, where $\mathcal{C}$ is compact on $\mathcal{H}$. Here the norm $\|\cdot\|_{\mathcal{H}}$ is defined by

$$
\|(v, q, \sigma, \lambda)\|_{\mathcal{H}}^{2}:=\|v\|_{M\left(\Omega_{F}\right)}^{2}+\|q\|_{P\left(\Omega_{F}\right)}^{2}+\|\sigma\|_{H^{3 / 2}\left(\Gamma_{0}\right) / C}^{2}+\|\lambda\|_{H^{1 / 2}\left(\Gamma_{0}\right) / C}^{2} .
$$

\section{Some computational results}

To conclude the paper, we present here some preliminary numerical results for a model problem in electromagnetic scattering by using a boundary-field equation method consisting of the Maxwell equations in the computational domain and one boundary integral equation, the MFIE on the artificial boundary $\Gamma_{0}$. We consider the electric scattering by a perfect conductor occupying a unit ball $\left\{(x, y, z): x^{2}+y^{2}+z^{2} \leq 1\right\}$. The incident wave propagates in the positive direction of $z$-axis

$$
\mathbf{E}^{\mathrm{i}}(x, y, z)=-\sqrt{\frac{\epsilon}{\mu}} e^{i k z} \hat{\mathbf{x}}, \quad \mathbf{H}^{\mathrm{i}}(x, y, z)=e^{i k z} \hat{\mathbf{y}},
$$


where $\hat{\mathbf{x}}, \hat{\mathbf{y}}$ are unit vectors in the direction of $x-$ and $y$ - axis, respectively. We are particularly interested in computing the far-field pattern or the scattering amplitude $\mathbf{H}_{\infty}(\hat{\mathbf{r}})$ or $\mathbf{H}_{\infty}(\theta, \phi)$ on the unit sphere from the resulting scattering field

$$
\mathbf{H}^{s}(\mathbf{x})=\frac{e^{i k|\mathbf{x}|}}{i k|\mathbf{x}|}\left(\mathbf{H}_{\infty}(\hat{\mathbf{r}})+O\left(\frac{1}{|\mathbf{x}|}\right)\right), \quad|\mathbf{x}| \rightarrow \infty .
$$

Here $\hat{\mathbf{r}}=\frac{\mathbf{x}}{|\mathbf{x}|}=(\sin \theta \cos \phi, \sin \theta \sin \phi, \cos \theta)(0 \leq \theta \leq \pi, 0 \leq \phi \leq 2 \pi)$ is a vector in the direction of $\mathbf{x}$ on the unit sphere. In the computation, the outer artificial boundary is chosen to be a sphere (with a radius of $a=1.4$ ). We triangulate the computational domain between the two concentric spheres and these triangulations also result in the partitions of both the scattering unit sphere and the artificial boundary sphere. In the computational domain, the Maxwell equations are discretized by employing the popular covolume scheme in computational electromagnetics (see, e.g., [12], [16]). These covolumne equations are then coupled with the discrete MFIE on $\Gamma_{0}$.

In Figure 1, we present two different meshes for the triangulations when the unit scattering sphere is divided into 128 and 512 patches. Typical components of the
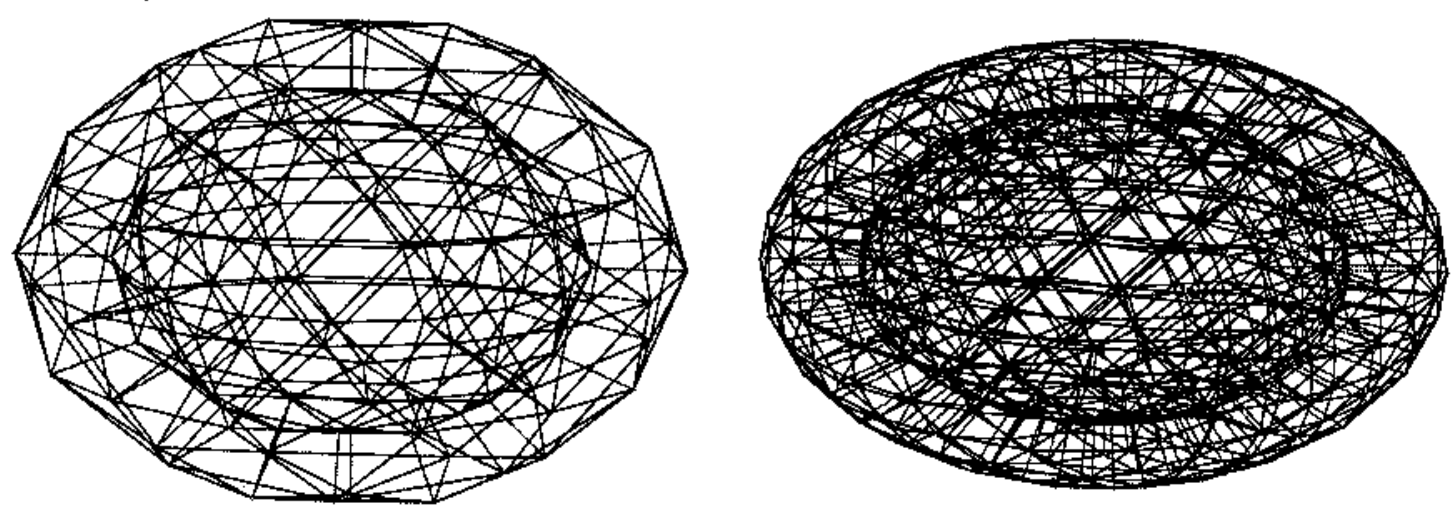

Figure 1: A computational domain and its triangulations.

far-field pattern $S_{1}(\theta)$ and $S_{2}(\theta)$ are computed. Here

$$
S_{1}(\theta):=\frac{H_{\phi}(\theta, \phi)}{\cos \phi}, \quad S_{2}(\theta):=\frac{H_{\theta}(\theta, \phi)}{\sin \phi}
$$

are independent of the angle $\phi$ and are related to the far-field pattern from the representation ([2])

$$
\mathbf{H}_{\infty}(\theta, \phi)=H_{\theta}(\theta, \phi) \hat{\boldsymbol{\theta}}+H_{\theta}(\theta, \phi) \hat{\boldsymbol{\phi}}
$$


where $\hat{\boldsymbol{\theta}}$ and $\hat{\phi}$ are the two orthogonal unit vectors on the tangent plane to the unit sphere at $\hat{\mathbf{r}}$. In the numerical experiments $S_{m}(\theta), m=1,2$, are computed at 39 different angles $\theta_{l}=l \pi / 39, l=0, \cdots, 38$. Figure 2 shows the results for $k a=0.5$ where the solid line and the circles represent the solution from the Mie series and the coupling scheme respectively. In this experiment, 128 triangular patches are used to partition the unit sphere. More numerical results and details concerning the
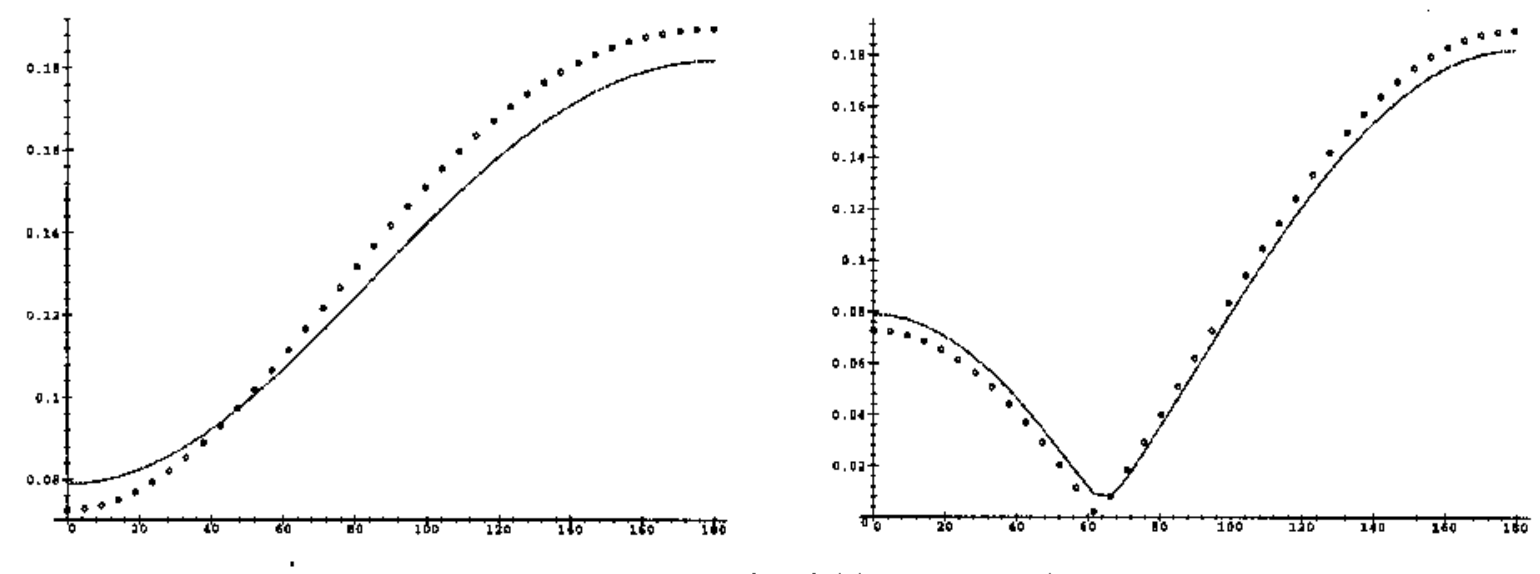

Figure 2: Plot of $\left|S_{1}(\theta)\right|$ and $\left|S_{2}(\theta)\right|$ for $k a=0.5$

combined methods of boundary element and covolume schemes in 3-D electromagnetic scattering will be available in a forthcoming paper [9].

\section{Acknowledgment}

This work was supported by the Air Force Office of Scientific Research, Air Force Material Command, USAF, under Grant F9620-96-1-10039. The views and conclusions contained herein are those of the author and should not be interpreted as necessarily representing the office policies or endorsements, either expressed or implied, of the Air Force Office of Scientific Research or the US government.

\section{References}

[1] H. Ammari and J.-C. Nédélec, Couplage éléments finis équations intégrals pour la résolution des équations de Maxwell en milieu hétérogéne, in Equations aux Dérivées Paertielles et Applications, pp. 1-15, Kluwer, 1998.

[2] J.J. Bowman, T.B.A. Senior and P.L.E. Uslenghi, Electromagnetic and acoustic scattering by simple shapes, North-Holland Publishing Company, 1969.

[3] M. Cessenat, Mathematical methods in electromagnetism, linear theory and applications, World Scientific Publishing Co. Pte. Ltd, 1996. 
[4] M. Costabel, Symmetric methods for coupling of finite elements and boundary elements, in Boundary element IV, 1, Compt. Mech., Southamption, 1987, pp. 411-420.

[5] G.N. Gatica and G.C. Hsiao, Boundary-field equation methods for a class of nonlinear problems, Pitman Research Notes in Mathematoics Series 331, Longman, 1995.

[6] D. Greenspan and P. Werner, A numerical method for exterior Dirichlet problem for the related wave equation, Arch. Rational Mech. Anall 23(1966), PP. 288316.

[7] G.C. Hsiao, The coupling of boundary element and finite element methods, ZAMM Z. angew. Mth. Mech. 70(1990), pp. T 493-T 503.

[8] G.C. Hsiao and R.E. Kleinman, Mathematical foundations for error estimation in numerical solutions of integral equations in electromagnetics, IEEE Trans on Antennas and Propagation, 45(1997), pp. 316-328.

[9] G.C. Hsiao and D.-Q. Wang, The combinded methods of boundary element and covolume schemes in 3D electromagnetic scattering, in preparation.

[10] A.de La Bourdonnaye, Some formulations coupling finite element and integral equation method for Helmholtz eqquation and electromagnetism, Numer. Math. 69(1995), pp. 257-268.

[11] R.C. MacCamy and S.P. Marin, A finite element method for exterior interface problems, Int. J. Math. and Math. Sci. 3(1990), pp. 311-350.

[12] R.A. Nicolaides and D.-Q. Wang, Convergence Analysis of a Covolume Scheme for Maxwell's Equations in Three Dimensions, Math. of Comp., to appear.

[13] A.H. Schatz, V. Thomée and W.L. Wendland, Mathmatical theory of finite and boundary element methods, DMV Seminar 15, Birkhäuser Verlag, 1990.

[14] J.A. Stratton and L.J. Chu, Diffraction theory of electromagnetic waves, Phy. Rev. 56(1939),pp. 99-107.

[15] W.L. Wendland, On asymptotic error estimates for the combined BEM and FEM. In: E. Stein and W.L. Wendland (eds): Finite element and boundary element techniques from mathematical and engineering point of view, CISM Lecture Notes 301, Udine, Springer-Verlag, Wein-New York 1988, pp. 273-333.

[16] K. Yee, Numerical solution of initial boundary value problems involving Maxwell's equations in isotropic media, IEEE Trans. Antennas and propagation, 16(1966), pp. 302-307. 